\title{
BMJ Open Clinical practice guidelines and principles of care for people with dementia: a protocol for undertaking a Delphi technique to identify the recommendations relevant to primary care nurses in the delivery of person- centred dementia care
}

To cite: Gibson C, Goeman D, Yates MW, et al. Clinical practice guidelines and principles of care for people with dementia: a protocol for undertaking a Delphi technique to identify the recommendations relevant to primary care nurses in the delivery of person-centred dementia care. BMJ Open 2021;11:e044843. doi:10.1136/ bmjopen-2020-044843

- Prepublication history for this paper is available online. To view these files, please visit the journal online (http://dx.doi org/10.1136/bmjopen-2020044843).

Received 17 September 2020 Revised 13 February 2021 Accepted 15 April 2021

Check for updates

(c) Author(s) (or their employer(s)) 2021. Re-use permitted under CC BY-NC. No commercial re-use. See rights and permissions. Published by BMJ.

For numbered affiliations see end of article.

Correspondence to

Caroline Gibson;

caroline.gibson@uon.edu.au

\section{ABSTRACT}

Introduction Nationally and internationally it is well recognised that dementia is poorly recognised and suboptimally managed in the primary care setting. There are multiple and complex reasons for this gap in care, including a lack of knowledge, high care demands and inadequate time for the general practitioner alone to manage dementia with its multiple physical, psychological and social dimensions. The primary care nurse potentially has a role in assisting the general practitioner in the provision of evidence-based dementia care. Although dementia-care guidelines for general practitioners exist, evidence on resources to support the primary care nurse in dementia care provision is scarce. The 'Australian Clinical Practice Guidelines and Principles of Care for People with Dementia' provides 109 recommendations for the diagnosis and management of dementia. This protocol describes a Delphi study to identify which of the 109 recommendations contained in these multidisciplinary guidelines are relevant to the primary care nurse in the delivery of person-centred dementia care in the general practice setting.

Methods and analysis Using a Delphi consensus online survey, an expert panel will grade each of the recommendations written in the 'Clinical Practice Guidelines and Principles of Care for People with Dementia' as high-to-low relevance with respect to the role of the primary care nurse in general practice. To optimise reliability of results, quality indicators will be used in the data collection and reporting of the study. Invited panel members will include Australian primary care nurses working in general practice, primary care nursing researchers and representatives of the Australian Primary Health Care Nurses Association, the peak professional body for nurses working in primary healthcare.

Ethics and dissemination This study has been approved by The University of Newcastle Human Research Ethics Committee (HREC) (H-2019-0029).

Findings will be published in a peer-reviewed journal and presented at scientific conferences.
Strengths and limitations of this study

- The Delphi consensus method is useful to solicit expert opinion and build knowledge in areas where limited evidence exists.

- Content validity is increased with selection of panel members with knowledge and interest in the roles of primary care nurses in general practice.

- Reliability of study findings are optimised using defined methodologic criteria for data collection and reporting in Delphi studies.

- The findings of this Delphi study will inform a qualitative interview study of Australian primary care nurses aimed at validating and refining the recommendations selected by the Delphi expert panel.

\section{INTRODUCTION}

Nationally and internationally, primary care, and in particular the general practitioner (GP), is responsible for the majority of screening, on-going management and care of people living with dementia (PLWD). ${ }^{12}$ However, it is well recognised that dementia is poorly recognised and suboptimally managed in the primary care setting. ${ }^{3}$ There are multiple and complex reasons for this gap in care, including a lack of knowledge, high care demands and inadequate time for the GP to manage dementia with its multiple physical, psychological and social dimensions. ${ }^{156}$

Primary care nurses (PCNs) are significant contributors in the delivery of primary care nationally and internationally ${ }^{7-10}$ and have a key role in supporting the work of the GP. ${ }^{11}$ It has been acknowledged that the PCN potentially has a role in supporting the GP in the better recognition and management 
of dementia in general practice. ${ }^{12-14}$ However, a review of recent literature shows that PCNs have limited knowledge in relation to the recognition of cognitive impairment and care planning for PLWD ${ }^{15}$ suggesting that they will need support if they are to have a role in assisting the GP to provide optimal care to PLWD.

Clinical practice guidelines aim to support health practitioners with best-practice decision-making ${ }^{16}$ thereby improve quality of care, patient outcomes and cost effectiveness. ${ }^{17} 18$ Although dementia care guidelines and pathways of dementia care delivered by GPs exist, there is little evidence on models of care or clinical practice guidelines that support the PCN in dementia care provision in general practice. ${ }^{19}$

The recently published 'Australian Clinical Practice Guidelines and Principles of Care for People with Dementia' ${ }^{20}$ (henceforth referred to as 'The Guidelines') provides 109 recommendations for the optimal diagnosis and management of dementia. The Guidelines are written for doctors, nurses, allied health and care workers who work with people in community, residential and hospital settings in Australia. ${ }^{20}$

The most effective clinical practice guidelines are those that are specific to the end-user and incorporate implementation frameworks. ${ }^{16} 2122$ Therefore, to optimise effectiveness of The Guidelines in supporting PCN delivery of dementia care, the 109 recommendations in The Guidelines need to be distilled down to those relevant to the PCN and barriers and enablers to the PCN implementation of these recommendations identified. This article describes a protocol for a Delphi technique that will identify the recommendations in The Guidelines that are perceived, by a panel with expertise in primary care nursing, as relevant to the PCN in the provision of evidence-based person-centred dementia care.

Findings from this Delphi process will inform the development of a future qualitative interview study with Australian PCNs to critically review the selected recommendations for delivering person-centred dementia care and identify barriers and enablers to their implementation in the general practice setting.

This study serves as an important first step to develop a primary care nursing model of care for the provision of dementia care in general practice, specific to the PCN role, and that also incorporates information on their implementation.

\section{METHODS AND ANALYSIS}

\section{Aim}

To identify which of the 109 recommendations contained in the 'Clinical Practice Guidelines and Principles of Care for People with Dementia' are relevant to the PCN in the delivery of person-centred dementia care within general practice.

\section{Design}

A Delphi consensus technique ${ }^{23}$ engaging an expert panel to reduce the number of recommendations to those applicable to $\mathrm{PCN}$ provision of dementia care will be used.

\section{Sample}

The expert panel is a purposive sample of people who have a minimum of 1 year's experience in research or clinical practice in the area of primary care nursing. Panel members invited to participate will include Australian practice nurses, primary care nursing researchers and representatives of the Australian Primary Health Care Nurses Association.

There is little agreement about the optimal size of the expert panel for a Delphi study ${ }^{23}$ and as described in Keeney $e t a l^{24}$ this decision is often based on funding, logistics of data handling and inclusion criteria. Jorm ${ }^{25}$ describes stability in findings with panels of around 20 or more members. This study will aim for 20 panel experts, assuming a 30\% rejection rate, 27 experts will be invited to participate in this study.

Participants will be anonymous to each other but not to the researcher. Panel members will be assured all information will be confidential and comply with the University of Newcastle research data security policies.

At the conclusion of the process, participants who have responded in each round will be offered the choice to remain anonymous, or receive acknowledgement in the publication for their participation.

\section{Recruitment}

Delphi participants will be invited to participate via email. The invitation to participate will include a personalised letter including a description of the study, its importance and the valuable contribution the panel member can make, the online Delphi process, expected time commitment and research contact details and a consent document. Written consent will be obtained by return email. After consent is provided, the first survey, round 1, of the Delphi will be sent to participants. Figure 1 describes the panel recruitment process.

\section{Enhancing response rate}

Strategies to aid the inclusion and commitment from panel members will include the following: electing panel members who are willing to participate and have knowledge of the relevant topic; communicating the ongoing importance of each individual panel member's contribution to the research process and that each round is constructed entirely on their response to previous rounds; limiting the number of rounds to reduce panel fatigue; quick turnarounds in data collection to enhance enthusiasm and maintain commitment, and consideration of panel members work and holiday schedules. ${ }^{23} 26$

\section{The Delphi online survey}

The Delphi online survey will be formatted using Research Electronic Data Capture. ${ }^{27}$ The online survey will list each 


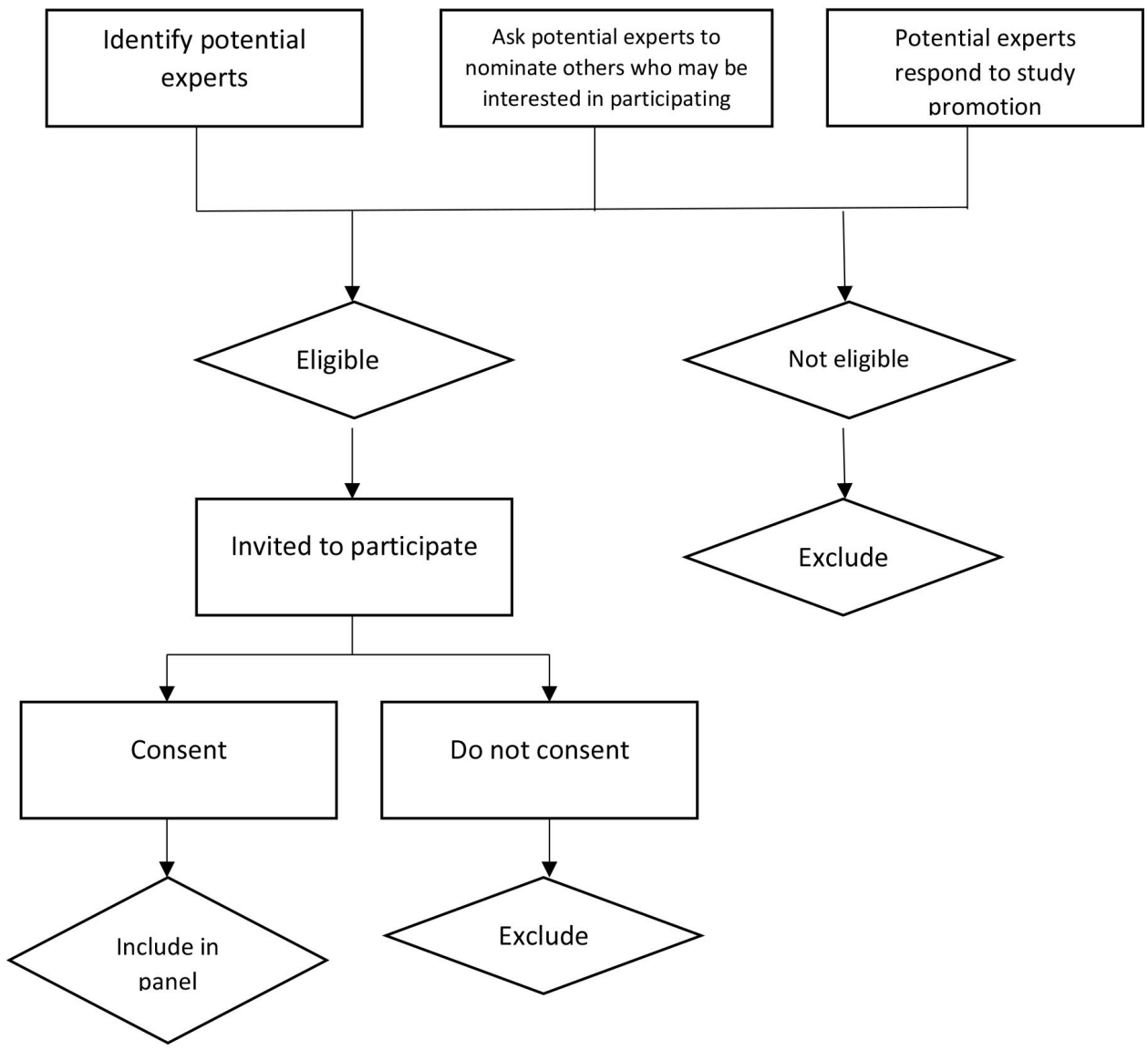

Figure 1 Expert panel recruitment process.

recommendation as written in The Guidelines. Each recommendation will be graded from high-to-low relevance using a five-point Likert scale in response to the question 'Is this recommendation relevant to the $\mathrm{PCN}$ in the provision of person-centred dementia care?' Suggestions for additional recommendations will not be sought as this study is limited to identifying those recommendations contained in the most recent Australian best-practice guidelines for dementia care that are relevant to the PCN.

\section{The survey rounds}

The round 1 survey will include all 109 recommendations and will take $<60 \mathrm{~min}$ to complete with subsequent rounds taking less time to complete as the number of recommendations for consideration become fewer.

Following the initial invitation, panel members will be given 10 days to provide their consent to participate and 2 weeks to respond between each round. Two reminders will be sent, the first at 1 week after the round is sent with the second reminder sent 2-3days prior to the due date of the round.

The Delphi process will run until consensus is met or for a maximum of three rounds as response exhaustion usually occurs within several rounds with 'busy' clinicians. ${ }^{24}$

\section{Returning data}

The responses from each round will be aggregated and fed back to the panel members anonymously in the next round. Group feedback will list the recommendations to be included, recommendations to be re-rated and recommendations to be excluded. Each panel member will also be provided with their individual responses and asked to reconsider the recommendations for which consensus has not been reached. Figure 2 illustrates the process of progression through the rounds and returning data.

\section{Consensus}

This study will use $75 \%$ agreement on the rating of relevance of the recommendation to the role of the PCN for determining consensus. Consensus refers to a certain percentage of the vote falling within a prescribed range ${ }^{23}$ or statistical analysis such as measures of central tendency mean and median and mode. In a systematic review on consensus in Delphi studies the median threshold, when specified, for determination of consensus was $75 \% .^{28}$ Those recommendations which are not excluded by consensus will be deemed as relevant.

\section{Data analysis}

The SPSS Statistics $25^{29}$ will be used for data analysis. A master code will be linked to each expert panel member. Each recommendation in The Guidelines will be a separate variable in SPSS. Frequencies run on entire dataset will provide the percentage of each overall response to each recommendation. The median will provide data on group opinion and the SD indicates level of agreement 


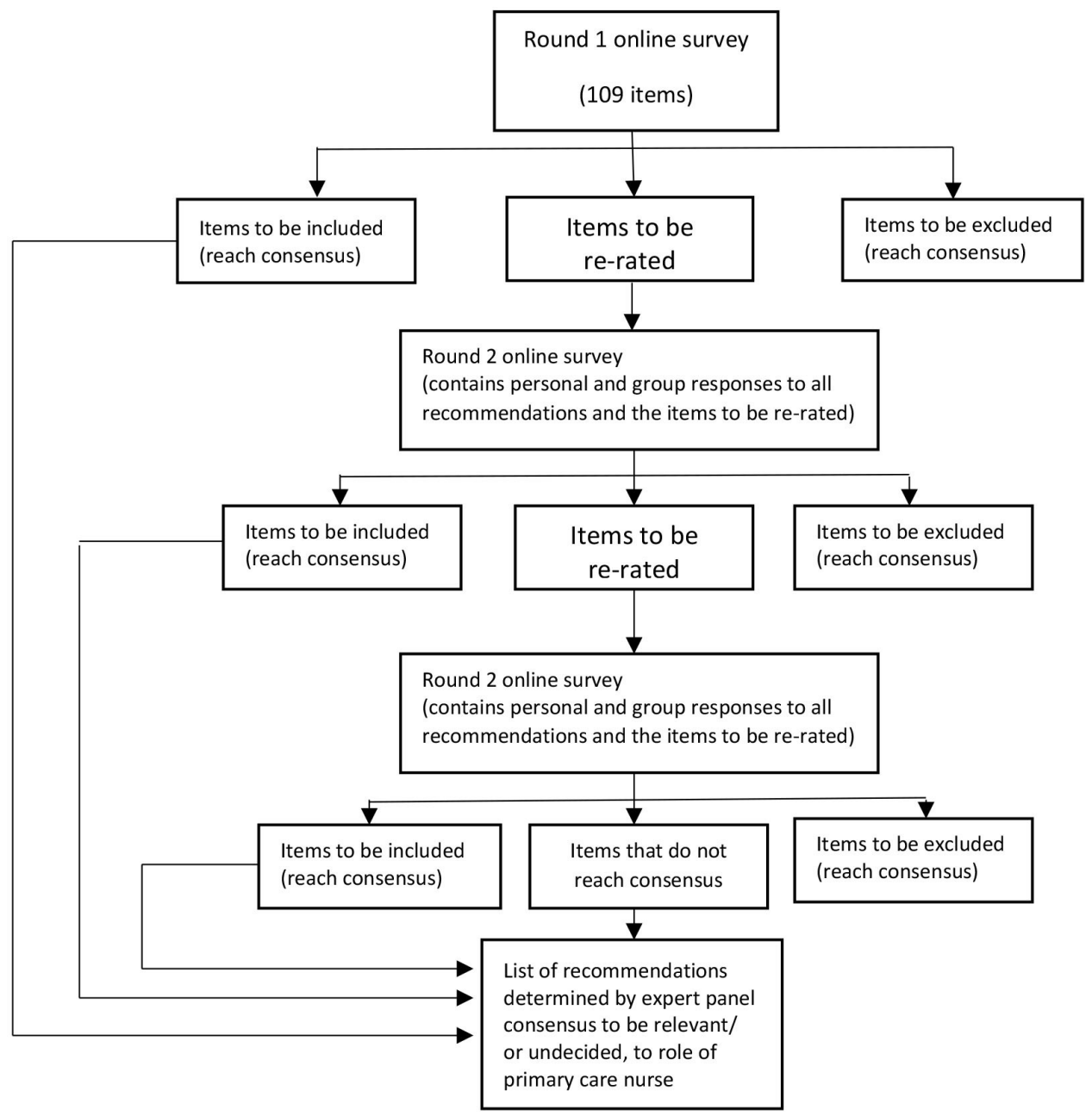

Figure 2 Progression through the rounds.

among the panel members. The mean will be used to rank the guidelines in order of relevance.

A descriptive analysis of the demographics of the expert panel will provide a profile overview.

\section{Quality and reporting}

This study will use quality indicators for reporting Delphi studies. The four quality indicators require a clear description of (1) the aim of the Delphi study including how it will address consensus, (2) participant inclusion and exclusion criteria, (3) the definition of consensus used and (4) the Delphi process with criteria for dropping items and determining when Delphi process to be stopped. $^{28}$

\section{Patient and public involvement}

Development of this study protocol did not involve direct public participation; however, consumers of dementia care were involved in the development of the Clinical Practice Guidelines and Principles of Care for People with Dementia ${ }^{20}$ which is central to this study.

\section{DISCUSSION}

This protocol describes the Delphi consensus technique that will be used to identify which of the 109 recommendations included in the 'Clinical Practice Guidelines and Principles of Care for People with Dementia' are agreed, by an expert panel, as relevant to the role of the PCN in the delivery of person-centred dementia care in the general practice setting.

The Delphi approach is appropriate as the research question does not lend itself to precise analytical techniques but can benefit from subjective judgements. ${ }^{28}$ This study design will overcome the significant constraints to surveying a large sample of PCNs. Namely, achieving a response rate to allow generalisability of the findings could be difficult with 109 items and the clear irrelevance of many items to the practice of PCNs in general practice.

The expert panel will be comprised of primary care nursing key stakeholders, including researchers, clinicians and representatives of primary care nursing associations. The criteria used for an 'expert' in this study is based on French $e t a l^{30}$ and is defined as an individual who has knowledge and experience about the subject of inquiry; is willing to express opinions about the subject of the inquiry and is able to express opinions about the subject of enquiry. 
Validated quality indicators for Delphi studies are lacking, ${ }^{24}$ therefore, in an attempt to mitigate this limitation, in this study we will use the Delphi methodologic criteria for reporting of Delphi studies proposed by Diamond $e t a l^{28}$

There are several expected limitations to using a Delphi method. Bias may result as selecting the experts cannot be random. ${ }^{24}$ Those who do participate are the most likely to be interested and not be truly representative of the larger population. ${ }^{24}$ However, content validity is increased by the use of participants who have knowledge and interest in the topic. ${ }^{31}$ Validity maybe compromised due to panel members changing highly relevant views in the face of a different view from the main body of the panel. ${ }^{32}$

Keeney $e t a l$ and Powell suggest that even when consensus exists, it does not indicate that an irrefutable fact has been achieved. ${ }^{23} 32$ The primary impact of achieving consensus is building new knowledge in an area of uncertainty using expert opinion. The findings of the Delphi consensus method can be enhanced with further research. ${ }^{32}$ Findings from this study will inform a qualitative interview study of Australian PCNs to determine which of the selected recommendations they perceive as applicable to their role in delivering person-centred dementia care and the feasibility of implementation in the general practice setting. Findings from this broader study will influence the development of an optimal primary care nursing model of care for people with dementia and support person(s) receiving care in the general practice setting.

\section{ETHICS AND DISSEMINATION}

This study has been approved by The University of Newcastle HREC (H-2019-0029).

Findings will be published in a peer-reviewed journal and presented at scientific conferences. The findings may also be presented to healthcare providers and consumers of dementia care in primary care settings via education forums, websites and social network services.

\section{Author affiliations}

${ }^{1}$ School of Medicine and Public Health, The University of Newcastle, Callaghan, New South Wales, Australia

${ }^{2}$ Department of Public Health and Medicine, The University of Newcastle, Callaghan, New South Wales, Australia

${ }^{3}$ Department of Medicine, Nursing and Health Sciences, Monash University, Clayton, Victoria, Australia

${ }^{4}$ Geriatric Medicine, Ballarat Health Service, Ballarat, Victoria, Australia

${ }^{5}$ Ballarat Clinical School, Deakin University, Ballarat, Victoria, Australia

${ }^{6}$ General Practice, University of Newcastle Australia, Callaghan, New South Wales, Australia

\section{Twitter Mark William Yates @YatesMarky}

Contributors CG: conceived the study. CG, DG, DP and MWY: contributed to the study design. CG and DG: drafted the manuscript, and MWY and DP: provided critical commentary on subsequent versions of the manuscript. All authors have read and approved the final version.

Funding This research contributes to a larger program of study being conducted by the Australian Community of Practice in Research in Dementia, which is funded by a Dementia Research Team Grant from the National Health and Medical Research Council. CG is supported by a University of Newcastle Postgraduate Research Scholarship from the Faculty of Health and Medicine.

\section{Competing interests None declared.}

Patient and public involvement Patients and/or the public were not involved in the design, or conduct, or reporting, or dissemination plans of this research.

Patient consent for publication Not required.

Provenance and peer review Not commissioned; externally peer reviewed.

Open access This is an open access article distributed in accordance with the Creative Commons Attribution Non Commercial (CC BY-NC 4.0) license, which permits others to distribute, remix, adapt, build upon this work non-commercially, and license their derivative works on different terms, provided the original work is properly cited, appropriate credit is given, any changes made indicated, and the use is non-commercial. See: http://creativecommons.org/licenses/by-nc/4.0/.

ORCID iD

Caroline Gibson http://orcid.org/0000-0002-3774-2601

\section{REFERENCES}

1 Spenceley SM, Sedgwick N, Keenan J. Dementia care in the context of primary care reform: an integrative review. Aging Ment Health 2015;19:107-20.

2 Islam MM, Parkinson A, Burns K, et al. A training program for primary health care nurses on timely diagnosis and management of dementia in general practice: an evaluation study. Int J Nurs Stud 2020;105:103550.

3 Koch T, Iliffe S. Dementia diagnosis and management: a narrative review of changing practice. Br J Gen Pract 2011;61:e513-25.

4 Strivens E, Craig D. Managing dementia-related cognitive decline in patients and their caregivers. Aust Fam Physician 2014;43:170-4.

5 Aminzadeh F, Molnar FJ, Dalziel WB, et al. A review of barriers and enablers to diagnosis and management of persons with dementia in primary care. Can Geriatr J 2012;15:85-94.

6 Heintz H, Monette P, Epstein-Lubow G, et al. Emerging collaborative care models for dementia care in the primary care setting: a narrative review. Am J Geriatr Psychiatry 2020;28:320-30.

7 Halcomb EJ, Davidson PM, Griffiths R, et al. Cardiovascular disease management: time to advance the practice nurse role? Aust Health Rev 2008;32:44-53.

8 Bauer L, Bodenheimer T. Expanded roles of registered nurses in primary care delivery of the future. Nurs Outlook 2017;65:624-32.

9 Keleher $\mathrm{H}$, Parker R, Abdulwadud O, et al. Systematic review of the effectiveness of primary care nursing. Int $J$ Nurs Pract 2009;15:16-24.

10 Norful A, Martsolf G, de Jacq K, et al. Utilization of registered nurses in primary care teams: a systematic review. Int J Nurs Stud 2017;74:15-23.

11 Sibbald B, Laurant MG, Reeves D. Advanced nurse roles in UK primary care. Med J Aust 2006;185:10-12.

12 Phillips J, Pond D, Goode S. Timely diagnosis of dementia: can we do better? A report for alzheimer's Australia. Canberra, 2011.

13 Manthorpe J, lliffe S, Eden A. Early recognition of dementia by nurses. J Adv Nurs 2003;44:183-91.

14 Trickey H, Turton P, Harvey I, et al. Dementia and the over-75 check: the role of the primary care nurse. Health Soc Care Community 2000;8:9-16.

15 Evripidou M, Charalambous A, Middleton N, et al. Nurses' knowledge and attitudes about dementia care: systematic literature review. Perspect Psychiatr Care 2019;55:48-60.

16 Gagliardi AR, Brouwers MC, Palda VA, et al. How can we improve guideline use? A conceptual framework of implementability. Implement Sci 2011;6:26.

17 Correa VC, Lugo-Agudelo LH, Aguirre-Acevedo DC, et al. Individual, health system, and contextual barriers and facilitators for the implementation of clinical practice guidelines: a systematic metareview. Health Res Policy Syst 2020;18:74.

18 Fischer F, Lange K, Klose K, et al. Barriers and strategies in guideline Implementation-A scoping review. Health Care 2016;4:36.

19 Gibson C, Goeman D, Pond D. What is the role of the practice nurse in the care of people living with dementia, or cognitive impairment, and their support person(s)?: a systematic review. BMC Fam Pract 2020;21:141.

20 Committee GA. Clinical practice guidelines and principles of care for people with dementia. Sydney Australia: NHMRC Partnership Centre 
for Dealing with Cognitive and Related Functional Decline in Older People, 2016.

21 Francke AL, Smit MC, de Veer AJE, et al. Factors influencing the implementation of clinical guidelines for health care professionals: a systematic meta-review. BMC Med Inform Decis Mak 2008;8:38.

22 Flodgren G, Hall AM, Goulding L, et al. Tools developed and disseminated by guideline producers to promote the uptake of their guidelines. Cochrane Database Syst Rev 2016;14.

23 Keeney S, Hasson F, McKenna H. The Delphi technique in nursing and health research. United Kingdom: Wiley Blackwell, 2011.

24 Keeney S, Hasson F, McKenna H. Consulting the oracle: ten lessons from using the Delphi technique in nursing research. J Adv Nurs 2006;53:205-12.

25 Jorm AF. Using the Delphi expert consensus method in mental health research. London, England: SAGE Publications, 2015: 887-97.

26 Boulkedid R, Abdoul H, Loustau M, et al. Using and reporting the Delphi method for selecting healthcare quality indicators: a systematic review. PLoS One 2011;6:e20476.
27 Harris PA, Taylor R, Thielke R, et al. Research electronic data capture (REDCap) - a metadata-driven methodology and workflow process for providing translational research informatics support. J Biomed Inform 2009;42:377-81.

28 Diamond IR, Grant RC, Feldman BM, et al. Defining consensus: a systematic review recommends methodologic criteria for reporting of Delphi studies. J Clin Epidemiol 2014;67:401-9.

29 Nie NH, Bent DH, Hull CH. SPSS: statistical package for the social sciences. New York: McGraw-Hill, 1975.

30 French P, Ho Y-Y, Lee L-S. A Delphi survey of evidence-based nursing priorities in Hong Kong. J Nurs Manag 2002;10:265-73.

31 Hasson F, Keeney S, McKenna H. Research guidelines for the Delphi survey technique. J Adv Nurs 2000;32:1008-15.

32 Powell C. The Delphi technique: myths and realities. J Adv Nurs 2003;41:376-82. 